\title{
EDUCACIÓN Médica
}

\section{Evolución de las universidades chilenas 1981-2004 ${ }^{1}$}

\author{
Ricardo Cruz-Coke $\mathrm{M}^{2}$. \\ The evolution of Chilean universities \\ from 1981 to 2004
}

In 1981, a supreme decree allowed the creation of private universities in Chile. As a consequence, 50 new universities were created in one decade, under the surveillance of the Council for Superior Education. This paper analyzes the evolution of this expansion process, that resulted in an admission of 370,000 students to 60 universities along the country, during 2004 . At the moment, $42 \%$ of the universities, designed as traditional, receive state financing and $58 \%$ are private. Twenty six percent are owned by the state, $52 \%$ are secular and $22 \%$ are confessional. The 25 traditional universities are complex organizations of a high academic level. New private universities are only devoted to teaching and some have obtained their autonomy. Some have improved the quality of their academic staff, perform research and impart doctorate degrees. However, most are small and with a limited academic staff. Traditional universities are stratified in a superior level. Eight private universities and some regional institutions, that are becoming complex and performing research activities, are stratified in a middle level. Two thirds of the private universities are in the lower level. The expansion of superior education is a sign of the social and cultural progress that Chile has experienced (Rev Méd Chile 2004; 132: 1543-9).

(Key Words: Education, medical, undergraduate; Schools, medical; Universities)

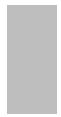

\footnotetext{
${ }^{1}$ Basado en una conferencia dictada en Sesión Ordinaria de la Academia Chilena de Medicina, el 7 de julio de 2004.

${ }^{2}$ Miembro de Número, Academia Chilena de Medicina, del Instituto de Chile.
}

$\mathrm{H}$ ace diez años, el Instituto de Chile me designó miembro del Consejo Superior de Educación, por lo cual durante cuatro años tuve la oportunidad de estudiar a fondo el proceso de reestructuración de la educación superior de nuestro país. Fruto de estos estudios, presenté dos conferencias sobre la descripción de las nuevas universidades privadas y sobre el tema de su acreditación ${ }^{1,2}$. En el debate, los académicos de esta corporación criticaron los cambios revolucionarios de nuestra educación superior y plantearon interrogantes sobre el futuro de las universidades chilenas. Después de siete años y entrando en un nuevo siglo, me ha parecido lógico estudiar la situación actual y evaluar las características de la evolución histónica de todas las universidades nacionales.

Ha sido mi intención abrir nuevamente un debate en el cual podamos aportar ideas para adaptarnos a la profunda y compleja revolución educacional que estamos viviendo. Describiré la actual situación y evolución de las universidades mediante la determinación de sus características y clasificación. Con esta información básica podremos evaluar más correctamente el futuro de las universidades tradicionales, su rol e influencia en el desarrollo y progreso de las nuevas universidades privadas. 
HistoRIA FUNDACIONAL DE LAS UNIVERSIDADES CHILENAS

Antes de la reestructuración de 1981 existían sólo 8 universidades en el país, que hoy se denominan "tradicionales". Dos de ellas eran estatales y las demás privadas con subvención estatal, pues cooperaban con la función educacional del Estado, de acuerdo con el concepto de Estado Docente y laico originado en el liberalismo y positivismo del siglo XIX (Tabla 1). Mediante el Decreto №2 de 3 de enero de 1981, el gobierno militar de la época, fragmentó las universidades estatales o públicas en sedes regionales y dictó una completa libertad de enseñanza superior para crear universidades privadas, con el mismo espíri- tu de don Abdón Cifuentes en 1873. Como resultado de este Decreto Supremo, se crearon las universidades derivadas de las tradicionales y 11 nuevas universidades privadas que serían sometidas a procesos de examinación por las universidades tradicionales (Tabla 2).

En 1986, se agruparon todas las universidades tradicionales y sus derivadas en un Consejo de 25 Rectores, que actuaba como una agencia de coordinación de derecho público que no afectaba la autonomía administrativa de sus componentes (Tabla 3).

En 1984, un grupo de académicos de la Universidad de Chile analizó críticamente la política educacional de las autoridades militares, que

\section{Tabla 1. Fundación de las universidades tradicionales}

\begin{tabular}{|clr|}
\hline Año de fundación & Nombre & Matrícula actual \\
\hline 1842 & Universidad de Chile & 23.548 \\
1888 & Pontificia Universidad Católica de Chile & 17.192 \\
1919 & Universidad de Concepción & 17.154 \\
1928 & Pontificia Universidad Católica de Valparaíso & 13.149 \\
1931 & Universidad Técnica Federico Santa María & 5.617 \\
1947 & Universidad Técnica del Estado (USACH) & 15.918 \\
1954 & Universidad Austral de Chile & 8.516 \\
1956 & Universidad Católica del Norte & $\mathbf{7 . 8 9 7}$ \\
& & 108.991 \\
\hline
\end{tabular}

Fuente: CSE, Indices 2004. El Mercurio.

Tabla 2. Fundación de las universidades privadas. G obierno militar, D FL N ㅇ, 1981

\begin{tabular}{|clcr|}
\hline Año de fundación & Nombre & Acreditación & Matrícula \\
\hline 1982 & Diego Portales & exam. & 9.135 \\
1982 & Gabriela Mistral & exam. & 2.306 \\
1982 & Central de Chile & exam. & 6.441 \\
1988 & Mayor & CSE & 12.708 \\
1988 & Finis Terrae & CSE & 2.717 \\
1988 & La República & CSE & 3.667 \\
1988 & Bolivariana & CSE & 1.903 \\
1989 & Andrés Bello & CSE & 14.584 \\
1989 & Adolfo Ibáñez & exam. & 2.858 \\
1989 & ARCIS & CSE & 4.562 \\
1989 & Las Américas & CSE & 14.855 \\
\hline
\end{tabular}

Fuente: CSE, Indices 2004. El Mercurio. 
afectaba gravemente a la Universidad de Chile, como la más antigua universidad tradicional ${ }^{3}$. En marzo de 1990, al término del gobierno militar, se dictó la Ley 18.962 Orgánica Constitucional de la Enseñanza (LOCE), que creó el Consejo Superior de Educación (CSE), destinado a supervigilar y acreditar a las nuevas universidades privadas. De este modo, en la década de los años 90 se fundaron otras 29 nuevas universidades (Tabla 4). En el proceso de acreditación fueron eliminadas seis de ellas y otras se fusionaron.

Después de un proceso de acreditación, estas nuevas instituciones pueden alcanzar la Autonomía, que es un derecho que concede el Estado, para que se rijan por sí mismas, con autonomía económica y administrativa. Adquieren los mismos derechos de las Universidades del Consejo de Rectores, salvo la subvención económica directa del Estado. Pero estas universidades privadas, por el Decreto DFL №4 de 1981, pueden recibir un Aporte Fiscal Indirecto (AFI) que beneficia a todas las universidades que matriculen en primer año a los 27.500 primeros puntajes de la Prueba de Aptitud Académica (PAA).

EVOLUCIÓN DE LA AUTONOMÍA DE LAS UNIVERSIDADES PRIVADAS

Al evaluar la situación del proceso de autonomía de las universidades privadas en 1997, informé a
Tabla 3. Fundación de las universidades derivadas. Consejo de Rectores. D FL N 22,1981

\begin{tabular}{|c|c|}
\hline $\begin{array}{l}\text { Año de } \\
\text { fundación }\end{array}$ & Nombre \\
\hline 1981 & $\begin{array}{l}\text { Antofagasta, Atacama, La Serena, } \\
\text { Valparaíso, La Frontera, Magallanes }\end{array}$ \\
\hline 1982 & Tarapacá, Católica de Temuco \\
\hline 1984 & Arturo Prat \\
\hline 1985 & Playa Ancha, Metropolitana \\
\hline 1988 & Bío-Bío \\
\hline 1991 & $\begin{array}{l}\text { Católica de Concepción, } \\
\text { Católica del Maule }\end{array}$ \\
\hline 1993 & Los Lagos, Tecnológica Metropolitana \\
\hline
\end{tabular}

Fuente: CSE, INDICES 2004. El Mercurio

esta Academia que el Consejo Superior de Educación (CSE) había concedido autonomía a 6 universidades privadas, cancelando la personalidad jurídica a 5 y prorrogando el plazo de concesión de autonomía a 10 de ellas. Por tanto, de las 31 universidades privadas en proceso de acreditación, sólo 6 de ellas habían alcanzado la autonomía, existiendo un fuerte proceso de selección.

En 2004, la escena ha cambiado radicalmente, pues ahora ya hay 30 universidades autónomas y están aún en proceso de acreditación otras 5. Por lo tanto, en Chile hay actualmente 55 universidades

Tabla 4. Fundación de las universidades privadas autónomas. Ley 18902, 1990, L.O.C.E.

\begin{tabular}{|clll|}
\hline $\begin{array}{c}\text { Año de } \\
\text { fundación }\end{array}$ & Nombre & \\
\hline 1990 & Adventista & Viña del Mar & Marítima \\
& Autónoma del Sur & Del Desarrollo & San Sebastián (Concepción) \\
& Bernardo O’Higgins & Del Pacífico & Santo Tomás \\
& Blas Cañas & SEK & \\
1991 & Los Andes & Mariano Egaña & \\
1992 & UNIACC & & \\
1993 & Vicente Pérez Rosales & & \\
1994 & Del Mar José S. Ossa & & \\
1997 & Iberoamericana & & \\
\hline
\end{tabular}

Fuente: CSE, Indices 2004. El Mercurio. 
autónomas, de las cuales 25 son las tradicionales y derivadas del Consejo de Rectores (Tabla 5).

$\mathrm{Al}$ parecer, después de dos décadas de evolución, se ha terminado de configurar el cuadro completo de las universidades nacionales del siglo XXI, con su gran diversidad cultural, que analizaremos al describir una clasificación general.

\section{CLASIFICACIÓN GENERAL DE LAS UNIVERSIDADES}

De acuerdo con los antecedentes reunidos en el proceso de fundación de las nuevas universidades chilenas en las dos últimas décadas, es posible identificar varias categonías determinadas por su naturaleza y antigüedad. En efecto, la división mayor es entre las tradicionales y no tradicionales y una subdivisión entre estatales, públicas, laicas y confesionales. La Tabla 6 , muestra la distribución de las distintas universidades, agrupadas de acuerdo a estas categorías.

De las 60 universidades chilenas enumeradas, 25 son tradicionales $(41,6 \%)$, en tanto que las no tradicionales son $35(58,3 \%)$. Las universidades estatales son $16(26,6 \%)$, y las religiosas o confesionales alcanzan a $13(21,7 \%)$. La mayoría, esto es 31 , son laicas $(51,4 \%)$. Sumando las laicas con las confesionales, resulta que hay 44 universidades privadas $(73,3 \%)$ que cubren la amplia mayoría de la educación superior nacional.

Respecto a su ubicación geográfica, las universidades han logrado distribuirse adecuadamente
Tabla 5. Evolución de autonomía de universidades 1997-2004

\begin{tabular}{|lrrrrr|}
\hline Autonomía & \multicolumn{2}{c}{1997} & \multicolumn{2}{c|}{2004} \\
CSE & $\mathrm{n}$ & $\%$ & \multicolumn{2}{c|}{$\mathrm{n}$} & $\%$ \\
\hline En acreditación & 26 & 40,6 & & 5 & 8,3 \\
En examinación & 7 & 11,0 & & 0 & 0 \\
Tradicional & 25 & 39 & & 25 & 41,6 \\
Privada autónoma & 6 & 9,3 & 30 & 50 \\
& 64 & 100 & 60 & 100 \\
\hline
\end{tabular}

en todo el territorio nacional. En Santiago hay 30, en el norte hay 14 y en la zona centro-sur el resto: 16. Mientras las universidades no tradicionales se concentran en Santiago, con 25 universidades, las tradicionales del Consejo de Rectores se distribuyen en casi todas las regiones, ya que en Santiago hay sólo 5 de ellas (Tabla 7). Por consiguiente el proceso de descentralización de la educación superior pública parece ser equilibrada.

En 2003, de acuerdo con el Directorio INDICE del Consejo Superior de Educación, la matrícula de pregrado de las 60 universidades chilenas alcanzaba a 370.686 estudiantes. Las 25 universidades tradicionales tenían 122.427 alumnos (33\%); las 17 derivadas de tradicionales 101.373 estudiantes (27,3\%); las 29 autónomas privadas 143.000 (38,6\%) y las 6 privadas en acreditación 3.880 estudiantes (1\%). En marzo de 2004, logró autonomía la Universidad Alberto Hurtado, que completó las 30 privadas autónomas, quedando 5 en acreditación.

Tabla 6. Clasificación general de las universidades chilenas en 2004

\begin{tabular}{|lrrrrr|}
\hline Categoría & Estatal & Laica & Confesional & Total & $\%$ \\
\hline Tradicional & & & & & \\
$\quad$ Original & 2 & 3 & 3 & 8 & 13,3 \\
Derivada & 14 & - & 3 & 17 & 28,3 \\
Subtotal & 16 & 3 & 6 & 25 & 41,6 \\
& & & & & \\
Privada & - & 23 & - & 23 & 38,3 \\
$\quad$ Laica & - & - & 7 & 7 & 11,0 \\
Confesional & - & 5 & - & 5 & 8,3 \\
Acreditación & & 28 & 7 & 35 & 58,3 \\
Subtotal & 16 & 31 & 13 & 60 & \\
Total & 26,6 & 51,4 & 21,7 & & 100 \\
$\quad \%$ & & & & & \\
\hline
\end{tabular}


ANÁLISIS COMPARATIVO DE LAS UNIVERSIDADES CHILENAS

Para comparar la calidad de las universidades se pueden usar los indicadores de excelencia académica aplicadas en nuestro estudio de la acreditación de 1998. Los mejores indicadores son: la matrícula de pregrado, el cuerpo docente de jornadas completas, los doctores, el aporte fiscal indirecto (AFI) y los marcadores de investigación científica y productividad. Hemos combinado estos indicadores en cuadros de correlación para comparar las universidades tradicionales con las mejores universidades privadas.

Al correlacionar el número de alumnos con AFI y la matrícula de pregrado, la Tabla 8 muestra como las universidades tradicionales ocupan los rangos más altos y las nuevas privadas los rangos más bajos, formando dos grupos con extremas

Tabla 7. D istribución geográfica de las universidades chilenas en 2004

\begin{tabular}{|c|c|c|c|}
\hline Región & Tradicional & Nuevas & Total \\
\hline 1. Tarapacá & 2 & - & 2 \\
\hline 2. Antofagasta & 2 & 1 & 3 \\
\hline 3. Atacama & 1 & - & 1 \\
\hline 4. Coquimbo & 1 & - & 1 \\
\hline 5. Valparaíso & 3 & 4 & 7 \\
\hline Santiago (Región Metropolitana) & 5 & 25 & 30 \\
\hline 6. Rancagua & 0 & 1 & 1 \\
\hline 7. Talca & 2 & 1 & 2 \\
\hline 8. Concepción & 3 & 2 & 5 \\
\hline 9. Temuco & 2 & 1 & 3 \\
\hline 10. Valdivia & 3 & 1 & 4 \\
\hline 12. Magallanes & 1 & - & 1 \\
\hline Total & 25 & 35 & 60 \\
\hline
\end{tabular}

Fuente: CSE, Indices 2004

Tabla 8. M ayores universidades chilenas

M atrícula y Aporte Fiscal Indirecto (AFI)

\begin{tabular}{|lccccc|}
\hline $\begin{array}{l}\text { AFI } \\
\text { Alumnos }\end{array}$ & $>2.000$ & $>5.000$ & $\begin{array}{c}\text { Matrícula } \\
>10.000\end{array}$ & $>15.000$ & $>20.000$ \\
\hline$>3000$ & & & PUC & U. Chile \\
$>2000$ & & USACH & \\
$>1000$ & & UVAL & UCV & CONCP & \\
& & UTSMA & & & \\
$>300$ & U. Andes & U. Austral & Mayor & & \\
& A. Ibáñez & Portales & A. Bello & & \\
& & Desarrollo & & & \\
& & & & & \\
& & & & & \\
\hline
\end{tabular}

PUC: Pontificia Universidad Católica de Chile. U. Chile: Universidad de Chile. USACH: Universidad de Santiago de Chile. UVAL: Universidad de Valparaíso. UCV: Pontificia Universidad Católica de Valparaíso. CONCP: Universidad de Concepción. UTSMA: Universidad Técnica Federico Santa María. U. Andes: Universidad de los Andes. U. Austral: Universidad Austral de Chile. Mayor: Universidad Mayor. A. Ibáñez: Universidad Adolfo Ibáñez. Portales: Universidad Diego Portales. A. Bello: Universidad Andrés Bello. Desarrollo: Universidad Del Desarrollo. 
diferencias. Las diferencias más significativas de estos dos grupos se revelan en la Tabla 9, al comparar el número de proyectos FONDECYT con el número de publicaciones anuales. Sólo aparecen en el cuadro las universidades tradicionales, pues las privadas autónomas no desarrollan investigación científica (con algunas excepciones). Sin embargo, donde las universidades privadas han logrado algunos avances es en la expansión del cuerpo docente. La Tabla 10, que relaciona el número de doctores con el número de docentes con jornada completa, muestra que las mayores privadas logran alcanzar el nivel de algunas universidades derivadas regionales. Pero en todos los cuadros comparativos, las grandes universidades tradicionales quedan separadas por distancias extremas.

A diferencia de las universidades tradicionales, las nuevas universidades privadas durante esta última década, han mantenido las características de no tener estructuradas carreras académicas estables, de mantener escasa proporción de docentes de jornada completa y con grados de doctores, para finalmente no tener prioridades en políticas de investigación científica y de innovación tecnológica. Sólo una pequeña minoría de ellas, ha comenzado a corregir estas deficiencias. Las seis mayores y mejores universidades privadas que hemos identificado tienen en conjunto una matrícula de 49 mil

\section{Tabla 9. M ayores universidades chilenas Investigación científica 2003}

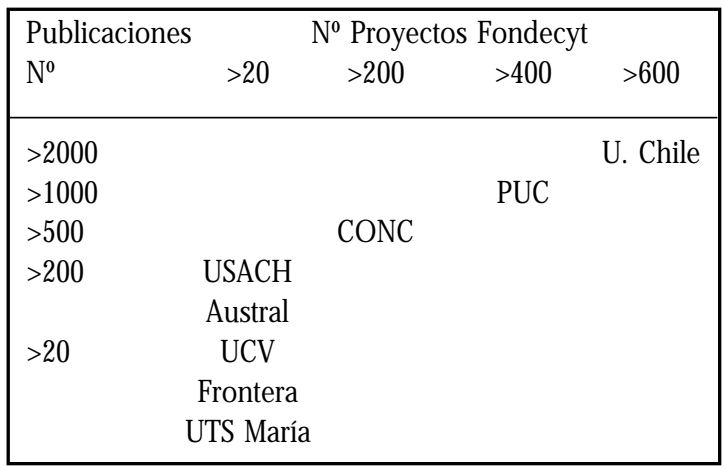

estudiantes, que representan el $34 \%$ del total de las 30 nuevas universidades privadas. Después de completar la etapa intermedia de desarrollo y obtener la autonomía, ellas están avanzando en competencia con las universidades tradicionales derivadas, en la obtención de los recursos estatales de apoyo a la docencia y a la investigación científica. Sin embargo, dos tercios de la matrícula de las nuevas universidades continúan en niveles de desarrollo docente, enfocadas a misiones específicas de sectores definidos de la sociedad en el campo social, empresarial, militar y religioso.

Tabla 10. M ayores universidades chilenas Académicos y D octores

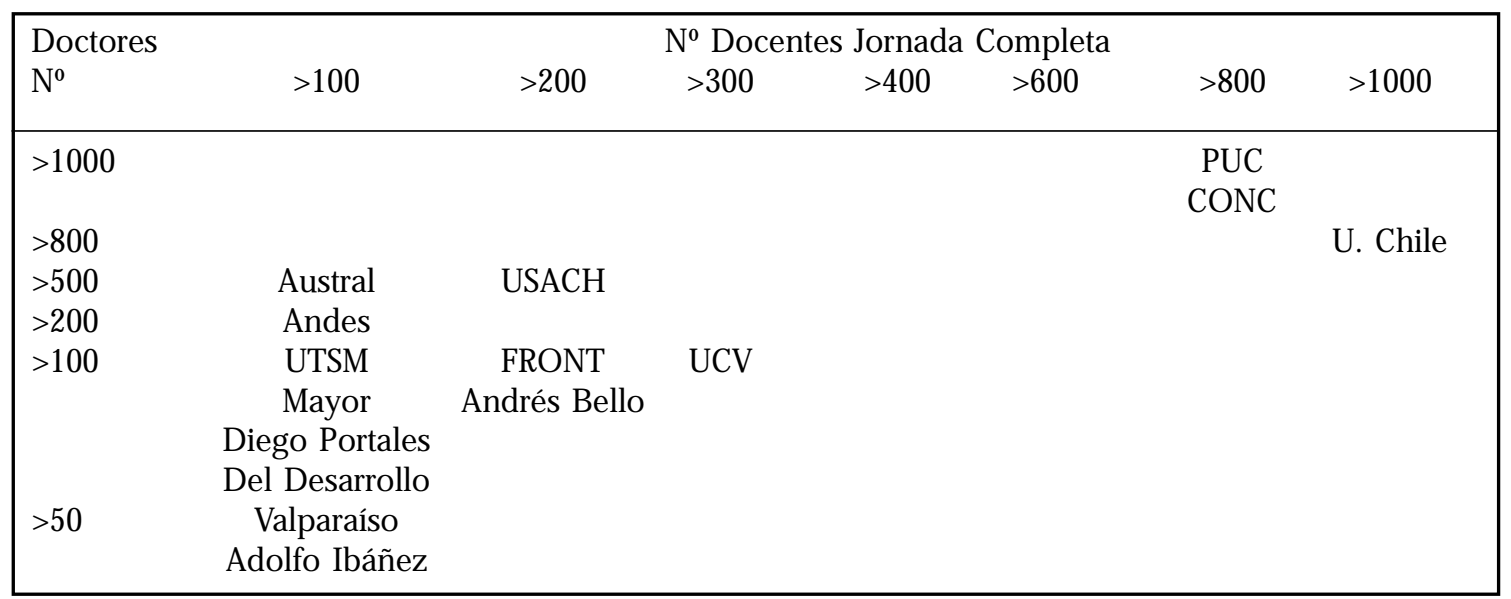


Durante estos últimos siete años, las mejores universidades privadas indicadas (Diego Portales, Mayor, Andrés Bello, Adolfo Ibáñez, Los Andes y Del Desarrollo) han efectuado considerables inversiones físicas y humanas, ampliando su extensión a las regiones del país y mejorando la calidad de la docencia, creando políticas de desarrollo científico y cursos de postgrado y postítulo, en competencia con las universidades tradicionales derivadas regionales. Están haciendo una positiva contribución ampliando la función de educación pública del Estado. Han alcanzado una altura de crucero en la evolución histórica de las universidades chilenas, pero debajo del alto nivel de las universidades tradicionales creadas en el siglo XIX.

\section{CONCLUSIÓN}

Falta aún tiempo para que el proceso de desarrollo de las nuevas universidades chilenas se estabilice, pero ya tenemos claro que ellas han alcanzado un importante grado de estratificación cualitativa, desde las grandes universidades centenarias del siglo XIX, hasta las pequeñas universidades docentes especializadas que tratan de representar modestamente las variadas culturas de la nación. Encabeza la lista de excelencia la Universidad de Chile, la más antigua, más grande y completa del país, que ha sobrevivido a la gran

\section{REFERENCIAS}

1. Cruz-Coke R. Las nuevas universidades privadas chilenas. Bol Acad Chil Med 1997; 34: 71-88.

2. CRUz-Coke R. Acreditación de autonomía de universidades privadas. Bol Acad Chil Med 1998; 35: 97-110. revolución cultural de fines del siglo $\mathrm{XX}^{3}$ y que hoy mantiene con sus renovaciones y reingenierías el alto nivel de sus cuadros académicos y el pluralismo del estado docente en la educación superior de la República. La escoltan la Pontificia Universidad Católica de Chile y algunas tradicionales de las regiones, que han logrado mantener su nivel de calidad.

En el nivel medio de desarrollo se ubican algunas de las universidades derivadas de regiones y las mejores universidades privadas autónomas señaladas, que necesitarán muchos años más para alcanzar el nivel de excelencia de las tradicionales. El resto de las nuevas universidades están ubicadas en el nivel inferior de evolución y deberán continuar progresando muchos años más, para completar el fortalecimiento de su autonomía con el apoyo de los mecanismos de autoevaluación y de acreditación y alcanzar así un lugar de excelencia en la comunidad académica nacional.

El aspecto más positivo de esta revolución universitaria es la formación de una gran diversidad de nuevas instituciones de educación superior, de tipo filosófico, religioso, político, económico y tecnológico. Ellas representan el amplio espectro cualitativo de la cultura nacional que, con la formación de una amplia base de clase dirigente académica, permitirá a la nación afrontar con confianza los desafíos de nuestra época.

3. Basso P, Cruz-Coke R, García G, Arancibia A, Díaz I, Lavados J et al. Hacia la normalización de la Universidad de Chile. Rev Méd Chile 1984; 112: 706-10. 